\title{
openheart A time-series study of percutaneous closure of patent foramen ovale: premature adoption?
}

\author{
Kian Nian Lew, ${ }^{1}$ Gianni D Angelini, ${ }^{2}$ William Hollingworth ${ }^{3}$
}

To cite: Lew KN,

Angelini GD, Hollingworth W. A time-series study of percutaneous closure of patent foramen ovale: premature adoption?. Open Heart 2016;3:e000313. doi:10.1136/openhrt-2015000313

Received 29 June 2015 Revised 25 August 2015 Accepted 13 October 2015

\section{CrossMark}

\footnotetext{
${ }^{1}$ Faculty of Medicine and Dentistry, University of Bristol, Bristol, UK ${ }^{2}$ Bristol Heart Institute, University Hospitals Bristol NHS Foundation Trust, Bristol, UK

${ }^{3}$ School of Social and Community Medicine, University of Bristol, Bristol, UK
}

Correspondence to Dr Kian Nian Lew; kl8321@my.bristol.ac.uk

\section{ABSTRACT}

Objectives: To evaluate the impact of National Institute for Health and Care Excellence (NICE) guidance in January 2005 and subsequent trial evidence on the adoption of percutaneous closure of patent foramen ovale (PCPFO).

Methods: A retrospective time series study was conducted using the Inpatient Hospital Episode Statistics (HES) England. A total of 3801 patients, aged $\geq 18$ and $\leq 60$ years, who had PCPFO from 1 April 2006 to 31 March 2012 in England. Percentage change annualised (PCA) in PCPFO procedure rates between initial NICE guidance and publication of trial results was analysed.

Results: Between Quarter 2, 2006 and Quarter 4 , 2009, 2163 PCPFO procedures were performed, with an increasing PCA of $48.4 \%$. The procedure rate peaked before the presentation of equivocal results from the first randomised controlled trial (RCT) in late 2010, and declined between Quarter 4, 2009 and Quarter 4, $2011(\mathrm{PCA}=-15.3 \%)$. Of more than 2300 patients recruited to three RCTs, only 71 were recruited in English hospitals.

Conclusions: PCPFO was rapidly adopted after the publication of initial NICE guidance despite the absence of RCT evidence of efficacy. Very few English patients participated in international RCTs of PCPFO, suggesting that NICE recommendations also failed to encourage the generation of RCT evidence.

\section{INTRODUCTION}

Patent foramen ovale is postulated to increase the risk of ischaemic stroke/transient ischaemic attack (TIA) through the paradoxical embolism mechanism which accounted for about $32 \%$ of ischaemic stroke/TIA. ${ }^{1}{ }^{2}$ Percutaneous closure of patent foramen ovale (PCPFO) is a relatively new day case interventional procedure that might reduce risk of the recurrence of stroke/TIA with low complication rates. Although rare, potential periprocedural major complications of PCPFO include pericardial tamponade $(0.2 \% ; 95 \%$ CI $0.1 \%$ to $0.2 \%)$, cerebrovascular events $(0.2 \%$; $95 \%$ CI $0.0 \%$ to $0.3 \%$ ), device embolism requiring

\section{KEY QUESTIONS}

What is already known about this subject?

- In January 2005, National Institute for Health and Care Excellence (NICE) guidance recommended that percutaneous closure of patent foramen ovale (PCPFO) could be used in the secondary prevention of stroke/transient ischaemic attack for audit or research purposes, despite lack of evidence from randomised controlled trials (RCTs).

What does this study add?

- PCPFO had been rapidly adopted with a percentage change annualised of $48.4 \%$ in the first 4 years since initial NICE guidance.

- RCTs of PCPFO have struggled to recruit, only a small number of English centres and patients contributed to RCTs.

- The increased adoption of PCPFO after initial NICE guidance despite the absence of RCT evidence suggests that NICE guidance on PCPFO encouraged premature adoption of PCPFO and may have stifled generation of RCT evidence.

How might this impact on clinical practice?

- Clinicians must be cautious in embracing nascent medical interventions until the safety and efficacy of the medical interventions are established.

- Greater clinicians' participation is needed in the recruitment of patients into RCT.

surgery $(0.1 \%$; $95 \%$ CI $0.0 \%$ to $0.2 \%)$ and retroperitoneal haematoma $(0.1 \% ; 95 \%$ CI $0.0 \%$ to $0.1 \%$ ). Long-term major complications include arrhythmias $(3.3 \% ; 95 \%$ CI $1.1 \%$ to $5.5 \%)$, cerebrovascular events $(1.5 \%$; $95 \%$ CI $1.0 \%$ to $2.0 \%$ ) and device thrombosis $(0.7 \%$; $95 \%$ CI $0.4 \%$ to $1.0 \%){ }^{3}$

In 2005, despite lack of randomised controlled trial (RCT) evidence, the National Institute for Health and Care Excellence (NICE) interventional procedures guidance recommended that $\mathrm{PCPFO}$ could be used in the secondary prevention of stroke/TIA with audit and further research of safety and 
efficacy. ${ }^{4}$ Three RCTs $^{5-7}$ published since 2010 comparing PCPFO with medical therapy found some evidence of reduction in the composite outcome of death or stroke/TIA, but the results were not statistically significant. Meta-analyses that combine stroke and TIA outcomes from these three RCTs find weak evidence that PCPFO is effective (eg, HR $=0.59 ; 95 \%$ CI 0.36 to 0.97$){ }^{8}$ Those that look at outcomes individually find weaker evidence (eg, HR $=0.62 ; 95 \%$ CI 0.34 to 1.11$)^{9}$ and tend to reach more negative conclusions. ${ }^{10}{ }^{11}$ Revised NICE guidance in 2013 recommended that PCPFO was suitable for routine National Health Service (NHS) use. ${ }^{12}$ Our study aims to evaluate the impact of NICE guidance in 2005 and dissemination of subsequent trial evidence from 2010 on the adoption of PCPFO.

\section{METHODS}

A time series study was conducted. Quarterly data on PCPFO procedure volumes from 1 April 2006 to 31 March 2012 was extracted from the English patient-level Hospital Episode Statistics (HES) database managed by the Health and Social Care Information Centre (HSCIC). HES is a routinely collected data set that records all day case or inpatient episodes of care provided to patients admitted to NHS hospitals and NHS funded patients treated in independent sector hospitals in England. A comprehensive synopsis of HES data is available at http://www.hscic.gov.uk/hes. All index cases were identified using the relevant (K16.5: Percutaneous transluminal closure of patent foramen ovale with prosthesis) Office of Population Census and Surveys (OPCS) procedure code V.4.3. ${ }^{13}$ To identify cases of PCPFO for secondary prevention of cryptogenic stroke/TIA, we excluded patients aged $<18$ years old and $>60$ years old as these age groups were excluded from the RCTs ${ }^{5-7}$ and are more likely to have PCPFO due to indications other than secondary prevention of stroke/TIA. In order to estimate procedure rate trends after March 2012, we used the publicly available data provided by the HSCIC $^{14}$ to calculate the average quarterly number of PCPFO procedures per year from 1 April 2006 to 31 March 2013. As these data are aggregated by HSCIC, we were not able to exclude patients based on age in this analysis. We calculated percentage change annualised (PCA) to summarise the diffusion of PCPFO procedures in England over time. ${ }^{15}$ We used Joinpoint trend analysis software to estimate the quarterly percentage change (QPC) in procedure counts and test for changes in that trend during the study period. ${ }^{16}$ The software fits a piecewise regression using weighted least squares with Poisson variance and a grid search method to identify between 0 and 3 joinpoints.

\section{RESULTS}

\section{Study sample}

From the individual patient data, we identified a total of 4388 PCPCO procedures between 1 April 2006 and 31
March 2012. A total of 587 patients aged $<18$ years old and $>60$ years were excluded, resulting in a final study cohort of 3801 patients. PCPFO was used in 1897 (49.9\%) men and $1903(50.1 \%)$ women with a mean age of $42.6(\mathrm{SD}=10.2)$ years (table 1$)$. A further 688 patients of all ages had PCPFO between 1 April 2012 and 31 March 2013.

\section{Trends in the adoption of PCPFO}

Between the start of the study period $(\mathrm{Q} 2,2006)$ and the peak in procedure volume (Q4, 2009), 2163 PCPFO procedures were performed, with a rapidly increasing PCA of $48.4 \%$ (figure 1 ). In the remainder of the study period (Q1, 2010 to Q1, 2012), 1638 PCPFO procedures were performed. Between the peak $(\mathrm{Q} 4,2009)$ and the nadir $(\mathrm{Q} 4,2011)$ after dissemination of initial trial results, the PCA declined $(\mathrm{PCA}=-15.3 \%)$. Analysis of aggregate data (without age exclusions) suggests that procedure rates fell further during 2012/2013 (figure 1). Data after March 2012 is an overestimate as it is based on national data which does not restrict PCPFO to those ages (18-60) thought most likely to be treated for cryptogenic stroke/TIA. Joinpoint regression identified two inflection points in procedure count trends. The initial rapid increase in procedure counts $(\mathrm{QPC}=23.9 ; 95 \%$ CI 0.8 to 54.6) attenuated after Q1 2007 until Q4, $2009(\mathrm{QPC}=4.2$; $95 \%$ CI 1.8 to 6.6 ) after which procedure rates declined ( $\mathrm{QPC}=-1.2 ; 95 \%$ CI 3.6 to 1.2 ).

The decline in procedure rates began before the initial conference presentation of results from the first RCT, CLOSURE 1 , in November $2010 .{ }^{17}$ CLOSURE 1 was published in March 2012, followed by RESPECT and PC-Trial in March 2013. These trials randomised more than 2300 patients; the only RCT (PC-Trial) that recruited from European centres, included 71 patients recruited from four English hospitals (Professor Bernhard Meier, 2014).

\section{DISCUSSION}

After the publication of NICE guidance on PCPFO in January 2005, the adoption of PCPFO rose rapidly

\begin{tabular}{|c|c|}
\hline Demographics & Number (\%) \\
\hline \multicolumn{2}{|l|}{ Sex } \\
\hline Male & $1897(49.91)$ \\
\hline Female & 1903 (50.07) \\
\hline \multicolumn{2}{|l|}{ Age group } \\
\hline $18-25$ & $255(6.71)$ \\
\hline $26-35$ & 681 (17.92) \\
\hline $36-45$ & 1257 (33.07) \\
\hline $46-60$ & 1609 (42.33) \\
\hline \multicolumn{2}{|l|}{ Race } \\
\hline White & 2979 (78.37) \\
\hline Others & 244 (6.42) \\
\hline Missing & 578 (15.21) \\
\hline
\end{tabular}


Figure 1 Trends in the adoption of percutaneous closure of patent foramen ovale (PCPFO), 20062013*. *Average quarterly procedures (Q2, 2006 to Q1, 2013) based on aggregate data on PCPFO procedures in patients of all ages; quarterly procedures (Q2, 2006 to Q1, 2012) based on individual patient data on PCPFO procedures in patients aged 1860 years only.

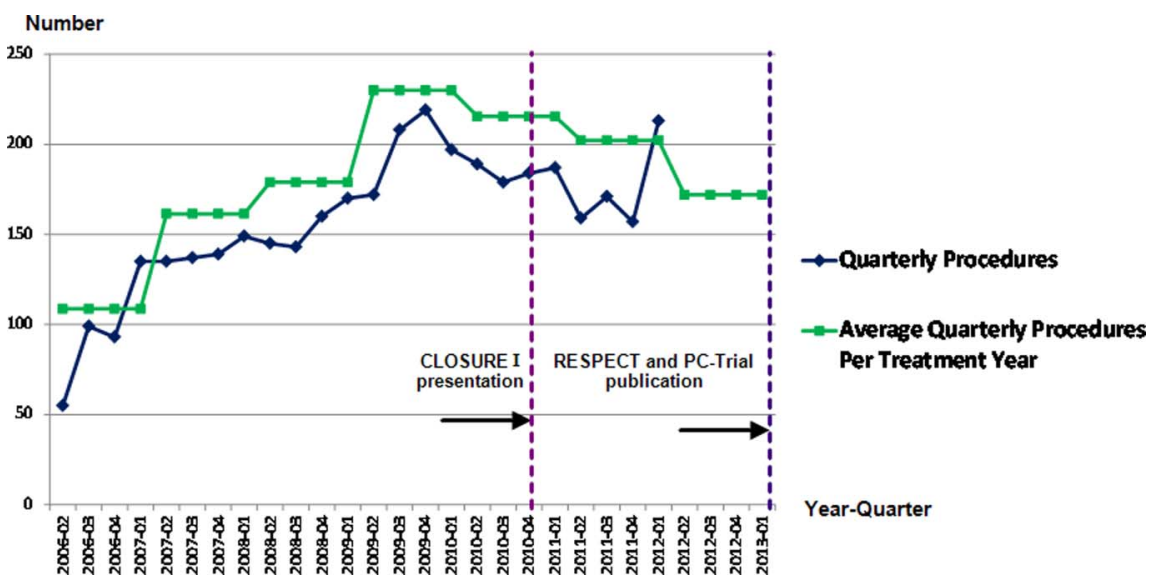

despite the lack of any RCT evidence of efficacy. More than 2000 procedures were performed before RCT results became available in November 2010. Despite NICE's recommendation that $\mathrm{PCPFO}$ be used in audit and with further research, only 71 patients from 4 English hospitals were randomised in the first 3 RCTs of PCPFO. The procedure rate peaked before the presentation of the equivocal results from the first RCT. ${ }^{17}$ Since then, the adoption of PCPFO had been falling until the end of the study period in March 2013. It remains unclear whether the current number of PCPFO procedures is appropriate, however, there is clear evidence that NICE guidance published in 2005 was associated with premature adoption of PCPFO before the efficacy was established and without stimulating sufficient engagement with RCTs.

Premature adoption can be defined as widespread technology uptake without adequate evidence of relative advantage, such as safety and cost-effectiveness. Adapted from the Rogers' S-shaped model of diffusion, ${ }^{18}$ the Balliol Collaboration suggest the peak rate of surgical innovation diffusion may occur once the first $10-20 \%$ of surgeons embrace the innovation and that the opportunity for formal assessment might then be lost. ${ }^{19}$ In this context, it is notable that enrolment to the three published PCPFO RCTs and other ongoing trials has lagged considerably, prompting a call for greater engagement among clinicians. ${ }^{20}$ Reasons for slow recruitment include 'off-label' use of PCPFO in patients considered too frail or with too many comorbidities for trial enrolment $^{21}$ as well as general clinician and patient barriers to $\mathrm{RCT}$ recruitment. ${ }^{22}$

It is likely that some patients treated with PCPFO in England were included in prospective registries and observational studies; therefore contributed to knowledge about adverse events and outcomes, although registries and observational studies are not well-suited for establishing treatment effectiveness. In the UK, there has been an ongoing effort to collect PCPFO complications and clinical outcome data through the establishment of the Central Cardiac Audit Database ${ }^{12}$ and recently the UK Percutaneous Patent Foramen Ovale Closure in adults (PFOC) registry. ${ }^{23}$ However, we are not aware of any peer-reviewed publications on PCPFO from these data sources. To the best of our knowledge, there had only been limited number of registries 24 and observational studies ${ }^{26-30}$ published from England, with a total of 899 patients of 3801 patients in our study cohort, highlighting the inefficiency in the enrolment of NHS patients into research studies.

In the case of PCPFO and many other medical innovations, premature adoption is encouraged by promising results from observational studies. ${ }^{31}$ For example, a meta-analysis study demonstrated that the HR of recurrent stroke in the three RCTs was 0.62 (95\% CI 0.34 to $1.11)$ compared to 0.23 (95\% CI 0.11 to 0.49$)$ in observational studies of PCPFO, ${ }^{9}$ suggesting bias and overestimation of the efficacy of PCPFO in early cohort studies. $^{32} 33$ Other case studies of potential premature adoption of cardiac interventional procedures include transcatheter aortic valve implantation (TAVI), ${ }^{34}$ coronary angiography and revascularisation. ${ }^{35}$

Our study has several limitations. First, we are unable to estimate the proportion of the patients who had PCPFO for the secondary prevention of ischaemic stroke/TIA because of the suboptimal coding of previous diagnoses in the HES database. ${ }^{36}$ Nevertheless, we believe that, after age criteria are applied, PCPFO for the secondary prevention of stroke/TIA constitutes the large majority of cases within our study. A large cohort study on 207 PCPFO cases showed that secondary prevention of cryptogenic stroke was the primary indication for PCPFO $(93 \%) .{ }^{37}$ Second, we are unable to show that there is a temporal increase of PCPFO adoption with NICE guidance implementation in 2005 due to unavailability of PCPFO OPCS procedure code before April 2006. However, we believe that NICE guidance had played a major role because it has been the intention of NICE interventional programme guidance to encourage and foster medical innovation. ${ }^{38}$ Third, we do not know to what extent improved coding accuracy, for example due to the introduction of payment by results hospital reimbursement, ${ }^{39}$ might have contributed to the increases in PCPFO procedure rates reported by hospitals. Nevertheless, we believe that this effect is limited as this reimbursement system was first introduced in 
2002. ${ }^{39}$ Finally, we cannot be certain whether the slow decline in procedure rates between 2011 and 2013 solely reflects caution among clinicians after the first equivocal RCT results or restrictions on funding introduced by healthcare commissioners in times of economic austerity. Although graphically the decrease of PCPFO adoption does not seem to be temporally related to the presentation of CLOSURE I results, we believe that the presentation of the CLOSURE I results had at least to some degree contributed to the decline, alongside with these limitations due to the multifactorial causes of PCPFO adoption.

\section{Implications and conclusion}

Premature adoption of unproven medical innovation is a form of low value care as unproven medical device with uncertain risk-to-benefit ratio ${ }^{40}$ offers little or no net clinical benefits in relative to the alternative treatments. ${ }^{41}$ It is an avoidable waste of healthcare resources and worse still could be harmful to patients. ${ }^{42}$ Recent scandals ${ }^{43}$ have prompted a change in the EU legislation on medical devices whereby high quality evidence is required for medium-risk and high-risk procedures before market approval. ${ }^{44}$ Clinicians must be mindful of embracing nascent medical technology until robust unbiased evidence on the safety and efficacy of new medical technology is established. We recommend the IDEAL framework in the evaluation of new medical innovations. ${ }^{45}$ Evidence generation should follow the hierarchy whereby the beginning of a RCT should mark the end of reliance from observational studies to provide evidence. Studies higher on the hierarchy of evidence, such as RCTs, should be given priority to avoid competition for patient recruitment. Clinicians understandably face challenges in RCT recruitment; support from policymakers and healthcare organisations are essential to overcome these barriers. ${ }^{22}$ In cases where RCTs are not possible due to rare events like in this case, observational studies on postmarketing surveillance of the medical devices are important to capture the outcome data to determine if the benefits of the medical device outweigh the risks. ${ }^{46}$

In conclusion, PCPFO was rapidly adopted after the publication of NICE guidance in 2005 despite the absence of RCT evidence of efficacy. Several thousand patients had the procedure in England before meta-analyses of three RCTs provided statistically weak evidence on the efficacy of PCPFO to reduce recurrent stroke/TIA. ${ }^{47}$ A meta-analysis study which includes the non-randomised studies demonstrated that there is an increasing net clinical benefits of PCPFO over time in terms of stroke/TIA recurrence and bleeding risks compared to the anticoagulant/antiplatelet therapies. ${ }^{48}$ Consequently, a personalised approach where the age of patient, comorbidities, interaction with other medications should be considered when deciding if PCPFO or medical therapy is suitable for a patient. NICE recommendations contributed to premature adoption of
PCPFO and failed to encourage the timely generation of RCT evidence. Further studies are required to investigate the factors leading to premature adoption of new medical innovation and how to prevent this phenomenon.

Acknowledgements The authors thank Professor Bernhard Meier and Dr Eveline Nüesch for providing the information on the number of participants recruited in the PC-Trial. HES data are Copyright 2013, re-used with the permission of The Health and Social Care Information Centre under data reuse agreement RU919. This paper was presented as a poster at the Second 'Preventing Overdiagnosis' conference, University of Oxford, 15-17 September 2014

Contributors KNL was responsible for the study concept and design, analysis and interpretation of data, drafting and revising the manuscript. GDA helped with data interpretation and provided critical comments on the manuscript and final draft. WH contributed to the study design, acquisition, analysis and interpretation of data, provided critical comments on the manuscript and the study supervision. All authors had full access to all of the data in the study and take responsibility for the integrity of the data and the accuracy of the data analysis. KNL is guarantor.

Funding This research study was supported by NIHR Bristol Cardiovascular Biomedical Research Unit and the British Heart Foundation (Grant Reference$\mathrm{CH} / 92027 / 7163)$. KNL was supported by a scholarship from the Public Service Department of Malaysia. WH is supported by funding from the MRC ConDuCT-II Hub (Collaboration and innovation for Difficult and Complex randomised controlled Trials In Invasive procedures-MR/K025643/1). The funding source was not involved in any part of the study design, data collection, analyses or drafting of the manuscript.

Competing interests None declared.

Ethics approval Analyses were conducted on pseudonymised data approved and provided by the Health \& Social Care Information Centre (HSCIC) to the University of Bristol, under a data re-use agreement (NIC 164132-C45WP-A1; RU919-A1).

Provenance and peer review Not commissioned; externally peer reviewed.

Data sharing statement No additional data are available.

Open Access This is an Open Access article distributed in accordance with the terms of the Creative Commons Attribution (CC BY 4.0) license, which permits others to distribute, remix, adapt and build upon this work, for commercial use, provided the original work is properly cited. See: http:// creativecommons.org/licenses/by/4.0/

\section{REFERENCES}

1. Davis D, Gregson J, Willeit $P$, et al. Patent foramen ovale, ischemic stroke and migraine: systematic review and stratified meta-analysis of association studies. Neuroepidemiology 2013;40:56-67.

2. Li L, Yiin GS, Geraghty OC, et al. Incidence, outcome, risk factors, and long-term prognosis of cryptogenic transient ischaemic attack and ischaemic stroke: a population-based study. Lancet Neurol 2015;14:903-13.

3. Abaci A, Unlu S, Alsancak Y, et al. Short and long term complications of device closure of atrial septal defect and patent foramen ovale: meta-analysis of 28,142 patients from 203 studies. Catheter Cardiovasc Interv 2013;82:1123-38.

4. NICE Interventional Procedure Guidance. Percutaneous closure of patent foramen ovale for the prevention of cerebral embolic stroke. National Institute for Health and Care Excellence, 2005. https://www. nice.org.uk/guidance/ipg109 (accessed 27 Jan 2015).

5. Furlan AJ, Reisman M, Massaro J, et al. Closure or medical therapy for cryptogenic stroke with patent foramen ovale. N Engl J Med 2012;366:991-9.

6. Carroll JD, Saver JL, Thaler DE, et al. Closure of patent foramen ovale versus medical therapy after cryptogenic stroke. $N$ Engl J Med 2013;368:1092-100.

7. Meier B, Kalesan B, Mattle HP, et al. Percutaneous closure of patent foramen ovale in cryptogenic embolism. N Engl J Med 2013;368:1083-91. 
8. Rengifo-Moreno P, Palacios IF, Junpaparp P, et al. Patent foramen ovale transcatheter closure vs. medical therapy on recurrent vascular events: a systematic review and meta-analysis of randomized controlled trials. Eur Heart J 2013;34:3342-52.

9. Capodanno D, Milazzo G, Vitale L, et al. Updating the evidence on patent foramen ovale closure versus medical therapy in patients with cryptogenic stroke: a systematic review and comprehensive metaanalysis of 2,303 patients from three randomised trials and 2,231 patients from 11 observational studies. Eurolntervention 2014;9:1342-9.

10. Kwong JS, Lam YY, Yu CM. Percutaneous closure of patent foramen ovale for cryptogenic stroke: a meta-analysis of randomized controlled trials. Int J Cardiol 2013;168:4132-8.

11. Nagaraja V, Raval J, Eslick GD, et al. Is transcatheter closure better than medical therapy for cryptogenic stroke with patent foramen ovale? A meta-analysis of randomised trials. Heart Lung Circ 2013;22:903-9.

12. NICE Interventional Procedure Guidance. Percutaneous closure of patent foramen ovale to prevent recurrent cerebral embolic events. National Institute for Health and Care Excellence, 2013. https://www. nice.org.uk/guidance/ipg472/evidence/ip2372-percutaneous-closureof-patent-foramen-ovale-to-prevent-recurrent-cerebral-embolicevents-topic-overview2 (accessed 23 Jun 2015).

13. Health \& Social Care Information Centre. Office of Population Censuses and Surveys (OPCS)-4 classifications of interventions and procedures.UK: The Stationary Office, 2006.

14. Health \& Social Care Information Centre. Hospital episode statistics, admitted patient care, England. Health \& Social Care Information Centre, http://www.hscic.gov.uk/ (accessed 17 Jul 2014).

15. Fay MP, Tiwari RC, Feuer EJ, et al. Estimating average annual percent change for disease rates without assuming constant change. Biometrics 2006;62:847-54

16. Kim HJ, Fay MP, Feuer EJ, et al. Permutation tests for joinpoint regression with applications to cancer rates. Stat Med 2000;19:335-51.

17. Crystal P. AHA: PFO Closure No Solution for Recurrent Stroke. Medpage Today website. Published on 15 November 2010. 2010. http://www.medpagetoday.com/MeetingCoverage/AHA/23392 (accessed 7 Jul 2014).

18. Rogers EM. Diffusion of innovations. Free Press, 2010

19. Barkun JS, Aronson JK, Feldman LS, et al. Evaluation and stages of surgical innovations. Lancet 2009;374:1089.

20. O'Gara P, Messe S, Tuzcu E, et al. Percutaneous device closure of patent foramen ovale for secondary stroke prevention: a call for completion of randomized clinical trials: a science advisory from the American Heart Association/American Stroke Association and the American College of Cardiology Foundation. Circulation 2009;119:2743.

21. Stackhouse KA, Goel SS, Qureshi AM, et al. Off-label closure during CLOSURE study. J Invasive Cardiol 2012;24:608-11.

22. Ross S, Grant A, Counsell C, et al. Barriers to participation in randomised controlled trials: a systematic review. J Clin Epidemiol 1999;52:1143-56.

23. National Institute for Cardiovascular Outcome Research. Health technology registries: percutaneous patent foramen ovale closure datasets and user guides. National Institute for Cardiovascular Outcome Research, 2014. https://www.ucl.ac.uk/nicor/registries/ pfo-closure/datasets (accessed 18 Jun 2015).

24. Thomson JD, Hildick-Smith D, Clift P, et al. Patent foramen ovale closure with the gore septal occluder. Catheter Cardiovasc Interv 2014:83:467-73.

25. Wöhrle J, Bertrand B, Søndergaard L, et al. PFO closuRE and Cryptogenlc StrokE (PRECISE) registry: a multi-center, international registry. Clin Res Cardiol 2012;101:787-93.

26. Thaman R, Faganello G, Gimeno J, et al. Efficacy of percutaneous closure of patent foramen ovale: comparison among three commonly used occluders. Heart 2011;97:394-9.
27. Mirzaali M, Dooley M, Wynne D, et al. Patent foramen ovale closure following cryptogenic stroke or transient ischaemic attack: long-term follow-up of 301 cases. Catheter Cardiovasc Interv 2015;86:1078-84

28. Ponnuthurai FA, van Gaal WJ, Burchell A, et al. Single centre experience with GORE-HELEX septal occluder for closure of PFO. Heart Lung Circ 2009:18:140-2.

29. Aral M, Mullen M. The Flatstent versus the conventional umbrella devices in the percutaneous closure of patent foramen ovale. Catheter Cardiovasc Interv 2015;85:1058-65.

30. MacDonald ST, Daniels MJ, Ormerod OJ. Initial use of the new GORE $®$ septal occluder in patent foramen ovale closure: implantation and preliminary results. Catheter Cardiovasc Interv 2013;81:660-5.

31. Kent DM, Kitsios GD. Percutaneous closure of patent foramen ovale: a near-perfect treatment ruined by careful study? Circ Cardiovasc Qual Outcomes 2012;5:414-15.

32. Ioannidis JP, Haidich AB, Pappa M, et al. Comparison of evidence of treatment effects in randomized and nonrandomized studies. JAMA 2001;286:821-30.

33. Kunz R, Oxman AD. The unpredictability paradox: review of empirical comparisons of randomised and non-randomised clinical trials. BMJ 1998;317:1185-90.

34. Van Brabandt H, Neyt M, Hulstaert F. Transcatheter aortic valve implantation (TAVI): risky and costly. BMJ 2012;345: 24-7.

35. Lange RA, Hillis LD. Use and overuse of angiography and revascularization for acute coronary syndromes. $N$ Engl J Med 1998:338:1838-9.

36. Burns E, Rigby E, Mamidanna R, et al. Systematic review of discharge coding accuracy. J Public Health (Oxf) 2012;34: $138-48$.

37. Nagpal SV, Lerakis S, Flueckiger PB, et al. Long-term outcomes after percutaneous patent foramen ovale closure. Am J Med Sci 2013;346:181-6.

38. Campbell B, Maddern G. Safety and efficacy of interventional procedures: scrutinising the evidence and issuing guidelines without stifling innovation. BMJ 2003;326:347.

39. Dixon J. Payment by results-new financial flows in the NHS: the risks are large but may be worth while because of potential gains. BMJ 2004;328:969.

40. Baker DW, Qaseem A, Reynolds PP, et al. Design and use of performance measures to decrease low-value services and achieve cost-conscious care. Ann Intern Med 2013;158:55-9.

41. Colla $\mathrm{CH}$. Swimming against the current-what might work to reduce low-value care? N Engl J Med 2014;371:1280-3.

42. Colla $\mathrm{CH}$, Morden NE, Sequist TD, et al. Choosing wisely: prevalence and correlates of low-value health care services in the United States. J Gen Intern Med 2015;30:221-8.

43. Horton R. Offline: the scandal of device regulation in the UK. Lancet 2012;379:204

44. Sorenson C, Drummond M. Improving medical device regulation: the United States and Europe in perspective. Milbank $Q$ 2014;92:114-50.

45. Ergina PL, Barkun JS, McCulloch P, et al. IDEAL framework for surgical innovation 2: observational studies in the exploration and assessment stages. BMJ 2012;346:f3011.

46. Eikermann M, Gluud C, Perleth M, et al. Commentary: Europe needs a central, transparent, and evidence based regulation process for devices. BMJ 2013:346:f2771.

47. Kitsios GD, Thaler DE, Kent DM. Potentially large yet uncertain benefits a meta-analysis of patent foramen ovale closure trials. Stroke 2013;44:2640-3.

48. Patti G, Pelliccia F, Gaudio C, et al. Meta-analysis of net long-term benefit of different therapeutic strategies in patients with cryptogenic stroke and patent foramen ovale. Am J Cardiol 2015;115:837-43. 